\title{
Prediction of pulmonary function in wheelchair users
}

\author{
R A Cooper PhD,${ }^{1,2}$ F D Baldini $\mathrm{PhD},{ }^{1}$ W E Langbein $\mathrm{PhD},{ }^{2} \mathrm{R} \mathrm{N}$ Robertson $\mathrm{PhD},{ }^{1,2}$ \\ P Bennett MS, ${ }^{1} \mathrm{~S}$ Monical MS ${ }^{1}$ \\ ${ }^{1}$ Human Engineering Laboratory, California State University, Sacramento, 95819-6019; \\ ${ }^{2}$ Rehabilitation Research \& Development Center, Veterans Affairs Medical Center, Hines, \\ IL 60141, USA.
}

As a result of the improved medical treatment of infectious diseases, these formerly leading causes of mortality in the United States have been supplanted in rank by chronic events as the major causes of death. The major causes include coronary artery disease, respiratory disease, and cancer. Static and dynamic pulmonary functions in the apparently healthy wheelchair user population were measured and then modelled using stepwise regression. One hundred and nine wheelchair users (97 males, 12 females) with paraplegia $(n=77)$ or quadriplegia $(n=32)$ gave informed consent and participated in this study. Subjects ranged from being Olympic caliber wheelchair marathon racers to those who live sedentary life styles. Subjects performed three slow vital capacity (SVC) tests, three forced vital capacity (FVC) tests, and three maximal voluntary ventilation (MVV) tests while seated in their standard wheelchair. The order of the pulmonary function tests was randomized. Subjects also completed two functional residual capacity (FRC) measurements. Analysis of variance revealed significant differences in several pulmonary functions based on gender (FEVC, $p=0.0001$, FEV1, $p=0.0001$, FEVC 25-75\%, $p=0.005$, PEF, $p=$ 0.002 , FIVC, $p=0.002, \mathrm{RV}, p=0.0001$, MVV, $p=0.0001, \mathrm{SVC}, p=0.001)$. The women's unforced prediction equations using age, height, weight did yield some significant correlations with predictions based upon ambulatory subjects. The men's unforced adapted prediction equations did reveal significant correlations with the validation group for FEVC $(r=0.66, p=0.007)$, FEV1 $(r=0.62, p=0.015)$, PIF $(r=0.95, p=0.015)$, MVV $(r=0.57, p=0.067)$, SVC $(r=0.69, p=0.019)$, and RV $(r=0.67, p=0.009)$. Pulmonary function in male wheelchair users should be predicted using equations which incorporate years with disability and level of impairment. Additional study is required to make any recommendations regarding women wheelchair users. However, study of pulmonary function in women should be made a priority. Pulmonary function is affected by the extent of physical impairment, and tends to decline as years with disability increase.

Keywords: wheelchair; respiratory functions; paraplegia; quadriplegia.

\section{Introduction}

Recent estimates show the prevalence of spinal cord injury (SCI) in the United States to be from 250,000 to 350,000 people, with annual incidents of new injuries at approximately $10,000 .^{1-4}$ As a result of the improved medical treatment of infectious diseases, these formerly leading causes of mortality have been supplanted in rank by chronic events as the leading causes of death in the United States, including coronary artery disease, respiratory disease, and cancer. It had been reported that $21 \%$ of the mortality rate among the SCI population is related to respiratory incidents which is not far behind cardiovascular incidents $(25 \%)$, the leading cause of mortality. ${ }^{1}$ Improvements in healthcare and continued research on pulmonary function will provide information for physicians, therapists and most 
importantly for individuals with mobility impairments which could increase the life expectancy of such individuals.

The increased numbers of wheelchair users, who are experiencing prolonged life present an important public health issue both in terms of resource allocation for long term management and of considerations for rehabilitation programs aimed at increasing functional aerobic work capacity. ${ }^{5.6} \mathrm{Be}-$ cause of their reduced levels of maximal aerobic work capacity resulting from loss of muscle function with concomitant physical deconditioning due to inactivity, the wheelchair user population appears to be especially disadvantaged for long term survival without a program of aerobic fitness training and testing.

It is well known that the level of physical impairment (spinal cord injury) affects the functions of the various aspects of the respiratory apparatus such as the intercostal muscles and diaphragm. Lower pulmonary functions have been documented in wheelchair users by several researchers. ${ }^{1.2 .7-12}$ More specifically, individuals with higher levels of impairment (quadriplegia) have poorer pulmonary functions than people with paraplegia.

Often the pulmonary function values for the wheelchair user are compared with normative tables obtained from the able bodied population ${ }^{13}$ which are based on gender, age and simple anthropometry. These tables are useful for initial evaluations of an individual's pulmonary functions. However, evaluation of pulmonary function among wheelchair users should be based on data collected from a wheelchair using population. ${ }^{1,14}$

The purpose of this project was to develop pulmonary function prediction equations based upon measured performances of apparently healthy wheelchair users. A secondary objective was to initiate the development of wheelchair user pulmonary function normative values including: forced expired vital capacity (FEVC), forced expired volume after one second (FEV1), forced expired vital capacity from 25 to $75 \%$ (FEVC 25-75\%), peak expiratory flow (PEF), forced inspired vital capacity (FIVC), peak inspiratory flow (PIF), resid- ual volume (RV), maximal voluntary ventilation (MVV), slow vital capacity (SVC), tidal volume (TV), and the ratio of FEV1/FEV.

\section{Methods}

\section{Subjects}

One hundred and nine wheelchair users (97 males, 12 females) with paraplegia $(n=77)$ or quadriplegia $(n=32)$ gave informed consent and participated in this study. The subjects' levels of chronic physical activity ranged from that of an Olympic caliber wheelchair marathon racer to that of a person leading a completely sedentary life style. Our subjects represent a balanced cross-section of chronic physical activity. Subjects were culturally and ethnically diverse and ranged in age from 17 to 69 years. Forty-nine percent of the men reported to be non smokers, whereas $75 \%$ of the women reported to be non smokers. Fiftyseven percent of the men who identified themselves as long term smokers reported that they had quit smoking. All subjects were free from illness at the time of participation in the study. This research was approved by the CSUS Committee for the Protection of Human Subjects.

\section{Experimental protocol}

Subjects performed three slow vital capacity (SVC) tests, three forced vital capacity tests (FVC), and three maximal voluntary ventilation (MVV) tests while seated in their standard wheelchair. The order of the pulmonary function tests was randomized. Spirometry was performed using either a dry rolling seal or a water seal spirometer (Vacumetrics, Ventura, CA or W.E. Collins, Braintree, MA). Spirometers were calibrated with a 31 syringe before testing. Other selected pulmonary functions were calculated from these tests. All tests were performed according to the recommendations for measurement of respiratory function of the American Thoracic Society.

\section{Statistical analysis}

Standard descriptive statistics were calculated for the pulmonary function data. 
These data were also expressed as percentiles for the men. There were insufficient numbers of women to present meaningful percentile values. A $2 \times 3$ ANOVA $(p<$ 0.05 ) was performed to determine the existence of any significant differences in pulmonary functions between men and women across disability groupings (upper level injury, C5-T3, middle level injury, T4-T10, lower level injury, T11 and lower). Sheffe's post hoc test was performed to determine within group differences with a significance level of $p<0.05$. Forward stepwise regression (forced and unforced) was used to develop models for predicting pulmonary function from age, height, weight, injury level, and years of injury. Due to the low number of female subjects all of their data were included for development of the female measurement models. Correlation analyses were performed for both males and females using prediction equations from Crapo et al. 15-17 Data from randomly selected male subjects whose pulmonary test results were not used to develop the male measurement models were acquired for the validation procedures. One way ANOVA $(p<0.05)$ was performed for each of the predicteds for the prediction and validation groups. When validating the models it was decided, a priori, that a type II error may be more serious than a type I error. Therefore a significance level of $p<0.10$ was chosen for regression analysis in an attempt to minimize the probability of committing a type II error, ie not detecting potentially significant correlations.

\section{Results}

Descriptive statistics for pulmonary function data for both men and women appear in Tables I, II and III, upper, middle and lower level injury, respectively. Analysis of variance revealed significant differences in several pulmonary functions based on gender (FEVC, $p=0.0001$, FEV1, $p=$ 0.0001 , FEVC $25-75 \%, p=0.005$, PEF, $p=0.002, \quad$ FIVC, $p=0.002, \quad \mathrm{RV}, \quad p=$ $0.0001, \quad \mathrm{MVV}, \quad p=0.0001, \quad \mathrm{SVC}, \quad p=$ $0.001)$. There were also differences in height $(p=0.0001)$, weight $(p=0.001)$ and years since injury $(p=0.047)$ between men and women. Because of these differences separate models were developed for men and women.

Between levels of injury there were significant differences in some pulmonary functions. Maximal voluntary ventilation measurements were significantly different across men in the upper and lower level injury $(p<0.05)$. Slow vital capacity data were different across men in the upper and middle groups $(p<0.05)$ as well as across men in the upper and lower groups $(p<0.05)$.

\section{Pulmonary function percentiles}

Percentile values for select pulmonary functions for men by impairment level are presented in Table IV. Percentile values were not presented for women as there were insufficient data to create meaningful cells. Table IV shows a gradual increase in pulmonary function as the level of impairment decreases. The range of values across the percentiles is indicative of the broad spectrum of people tested.

\section{Regression models for predicting pulmonary function}

Forward stepwise regression was used to determine prediction models for pulmonary functions of men and women wheelchair users based upon age, height, weight, injury level, and years of injury. Typically pulmonary function prediction equations that have been developed for ambulatory individuals are based upon age, height and weight. Therefore these measurements were also examined as possible independent variables for predicting pulmonary function of men and women wheelchair users (Table V). Level of impairment was not used in this analysis. In this analysis age had the highest and most frequent partial correlation coefficient for men, whereas height appeared most often for women. Age had a negative partial correlation coefficient in nearly all equations in which it appeared for men and women. For men the equation for RV had the highest correlation $(r=0.42)$, whereas for the women the FEVC equation had the highest correlation $(r=0.85)$, (Table V). Significant partial correlations were found 
Table I Pulmonary function measurements for men and women wheelchair users with upper level impairments (laminae C5-T3)

\begin{tabular}{lccccc}
\hline & Mean & Std Dev & Min & Max & No \\
\hline Men & & & & & \\
Age (yrs) & 33.3 & 8.24 & 17 & 51 & 29 \\
Years injured (yrs) & 11.2 & 5.99 & 4 & 24 & 20 \\
Height (cm) & 179.5 & 8.5 & 160.6 & 194.3 & 29 \\
Weight (kg) & 71.5 & 11.63 & 48.9 & 102.7 & 29 \\
FEVC (l) & 4.04 & 1.051 & 2.28 & 6.56 & 29 \\
FEV1 (l) & 3.24 & 0.865 & 1.81 & 5.09 & 29 \\
FEVC 25-75\% (l) & 3.63 & 1.4 & 1.23 & 5.35 & 13 \\
PEF (l/min) & 6.12 & 2.183 & 2.19 & 11.13 & 22 \\
FIVC (l) & 4.2 & 1.036 & 2.53 & 6.74 & 13 \\
PIF (l/min) & 4.47 & 1.817 & 2.42 & 8.31 & 19 \\
RV (l) & 2.13 & 0.737 & 0.99 & 3.9 & 19 \\
MVV (l/min) & 125.5 & 34.33 & 73.2 & 183.6 & 22 \\
SVC (l) & 3.88 & 0.739 & 2.8 & 5.2 & 13 \\
TV (l) & 0.91 & 0.374 & 0.4 & 1.8 & 13 \\
FEV1/FVC & 0.81 & 0.1 & 0.59 & 1 & 29 \\
Women & & & & & \\
Age (yrs) & 37.8 & 10.35 & 26 & 52 & 5 \\
Years injured (yrs) & 17 & 12.15 & 4 & 32 & 5 \\
Height (cm) & 166.1 & 6.39 & 157.5 & 175.3 & 5 \\
Weight (kg) & 62.2 & 21.07 & 39.1 & 94.5 & 5 \\
FEVC (l) & 2.91 & 0.607 & 2.08 & 3.62 & 5 \\
FEV1 (l) & 2.48 & 0.584 & 1.63 & 2.98 & 5 \\
FEVC 25-75\% (l) & 2.63 & 0.856 & 2.02 & 3.23 & 2 \\
PEF (l/min) & 4.68 & 0.389 & 4.4 & 4.95 & 2 \\
FIVC (l) & 2.89 & 0.453 & 2.57 & 3.21 & 2 \\
PIF (l/min) & 3.42 & & 3.42 & 3.42 & 1 \\
RV (l) & 1.55 & 0.561 & 0.93 & 2.26 & 4 \\
MVV (l/min) & 48 & 25.03 & 30.3 & 65.7 & 2 \\
SVC (l) & 2.75 & 0.495 & 2.4 & 3.1 & 2 \\
TV (l) & 1.05 & 0.212 & 0.9 & 1.2 & 2 \\
FEV1/FVC & 0.85 & 0.212 & 0.78 & 0.94 & 5 \\
\hline
\end{tabular}

for the pulmonary function measures FEVC, FEV1, PIF, RV, and MVV for men. For women significant partial correlations between the selected independent variables were found for FEVC, FEV1, FEVC $25-75 \%$, PEF, FIVC, and PIF.

Forced stepwise regression equations for men and women were also developed based upon age (years), height (centimeters) and weight (kilograms), independent of level of impairment (Table VI). Again, for men the equation for residual volume (RV) had the highest correlation $(r=0.46)$, whereas for women the FIVC equation had the highest correlation $(r=0.87)$ (Table VI). The equations for women all had higher correlations than the equations for men. However, there were substantially fewer women than men. Age had a negative partial correlation with pulmonary volumes (FEVC, FEV1, SVC, MVV) and flows (PEF, PIF) for men. For women, age had negative partial correlations with pulmonary volumes (FEVC, FEV1, FVC 25-75\%, FIVC, RV, MVV, SVC) and flow (PIF).

The equations developed to predict pulmonary functions of wheelchair users based upon age, height and weight did not yield substantial correlations. In addition, analysis of variance indicated that for some variables there is some dependence upon injury (impairment) level. Because age is an important factor in predicting the pulmonary function of ambulatory individuals, it 
Table II Pulmonary function measurements for men and women wheelchair users with middle level impairments (laminae T4-T10)

\begin{tabular}{lccccc}
\hline & Mean & Std Dev & Min & Max & No \\
\hline Men & & & & & \\
Age (yrs) & 35.7 & 9.73 & 22 & 60 & 54 \\
Years injured (yrs) & 9.6 & 4.78 & 3 & 22 & 35 \\
Height (cm) & 179.4 & 7.06 & 162.6 & 193 & 54 \\
Weight (kg) & 77 & 15.69 & 54.2 & 125.8 & 54 \\
FEVC (l) & 4.39 & 1.051 & 1.7 & 8.9 & 54 \\
FEV1 (l) & 3.59 & 0.742 & 1.55 & 5.17 & 54 \\
FEVC 25-75\% (l) & 4.75 & 1.667 & 1.97 & 9.84 & 22 \\
PEF (l/min) & 7.37 & 2.372 & 3.48 & 13.48 & 47 \\
FIVC (l) & 4.87 & 1.151 & 3.06 & 8.59 & 21 \\
PIF (l/min) & 5.49 & 2.246 & 1.58 & 9.81 & 38 \\
RV (l) & 2.25 & 0.57 & 0.87 & 3.5 & 33 \\
MVV (l/min) & 149 & 40.73 & 62 & 256 & 48 \\
SVC (l) & 4.1 & 0.679 & 3 & 5.58 & 29 \\
TV (l) & 1.1 & 0.446 & 0.4 & 2.4 & 29 \\
FEV1/FVC & 0.83 & 0.117 & 0.35 & 1 & 54 \\
Women & & & & & \\
Age (yrs) & 35.8 & 3.11 & 32 & 39 & 5 \\
Years injured (yrs) & 14.6 & 5.73 & 10 & 24 & 5 \\
Height (cm) & 163.3 & 21.71 & 125.7 & 177.8 & 5 \\
Weight (kg) & 59.6 & 20.32 & 32.3 & 82.8 & 5 \\
FEVC (l) & 3.08 & 1.344 & 1.13 & 4.36 & 5 \\
FEV1 (l) & 2.39 & 0.884 & 0.99 & 3.41 & 5 \\
FEVC 25-75\% (l) & 2.82 & 0.86 & 1.86 & 3.52 & 3 \\
PEF (l/min) & 5.3 & 1.557 & 3.5 & 6.26 & 3 \\
FIVC (l) & 3.74 & 0.609 & 2.52 & 4.37 & 3 \\
PIF (l/min) & 4.26 & 1.002 & 3.18 & 5.16 & 3 \\
RV (l) & 0.93 & 0.412 & 0.42 & 1.46 & 5 \\
MVV (l/min) & 90.5 & 54.19 & 30.4 & 135.7 & 3 \\
SVC (l) & 3.42 & 0.886 & 2.6 & 4.36 & 3 \\
TV (l) & 0.95 & 0.132 & 0.8 & 1.05 & 3 \\
FEV1/FVC & 0.81 & 0.124 & 0.59 & 0.89 & 5 \\
\hline
\end{tabular}

can be argued that, if a person acquires a disability sometime during their lifetime, the years since injury could contribute to predicting their pulmonary function.

Unforced stepwise regression analysis using age in years, height in centimeters, weight in kilograms, years injured in years, and injury level were used as independent variables to generate the equations for Table VII. Level of injury was numerically scaled by assigning a number based upon anatomical location of lesion, ie each vertebra was assigned a progressively higher number starting with the first cervical vertebra. For men the years injured (YI) and injury level (IL) had the strongest partial correlation coefficients in nearly all of the equations. Years injured was most often negatively correlated with pulmonary function, whereas injury level was most often positively correlated. Residual volume (RV) was best predicted with height and weight. The results from Table $\mathrm{V}$ and Table VII differ primarily for two reasons: (a) the independent variables were different which altered the partial correlations, and (b) the subject pool used to develop the equations was different because complete data were not available for all subjects. The stepwise regression analysis of male subject data revealed significant prediction equations for FEVC, FEV1, PEF, FIVC, PIF, RV, 
Table III Pulmonary function measurements for men and women wheelchair users with lower level impairments (laminae T11 and lower)

\begin{tabular}{lccccc}
\hline & Mean & Std Dev & Min & Max & No \\
\hline Men & & & & & \\
Age (yrs) & 37.3 & 11.29 & 20 & 69 & 35 \\
Years injured (yrs) & 15.8 & 13.68 & 2 & 48 & 17 \\
Height (cm) & 176.6 & 17.2 & 106.7 & 203.2 & 35 \\
Weight (kg) & 77 & 18.32 & 42.1 & 131.7 & 35 \\
FEVC (l) & 4.57 & 0.877 & 2.88 & 6.65 & 35 \\
FEV1 (l) & 3.79 & 0.657 & 2.57 & 4.99 & 35 \\
FEVC 25-75\% (l) & 4.88 & 0.763 & 3.9 & 6.22 & 9 \\
PEF (l/min) & 8.65 & 2.169 & 4.29 & 12.95 & 30 \\
FIVC (l) & 4.82 & 0.946 & 3.43 & 6.49 & 9 \\
PIF (l/min) & 5.73 & 2.594 & 1.32 & 12.15 & 23 \\
RV (l) & 1.93 & 0.618 & 0.9 & 3.23 & 17 \\
MVV (l/min) & 165.6 & 36.98 & 102.8 & 251 & 30 \\
SVC (l) & 4.77 & 0.844 & 3.4 & 6.7 & 12 \\
TV (l) & 1.16 & 0.367 & 0.73 & 2.1 & 12 \\
FEV1/FVC & 0.83 & 0.076 & 0.67 & 0.97 & 35 \\
Women & & & & & \\
Age (yrs) & 36.6 & 12.8 & 24 & 60 & 7 \\
Years injured (yrs) & 19.6 & 14.37 & 3 & 46 & 7 \\
Height (cm) & 161.4 & 13.32 & 139.7 & 175.2 & 7 \\
Weight (kg) & 61.4 & 7.14 & 52.9 & 70.6 & 7 \\
FEVC (l) & 3.33 & 0.861 & 2.23 & 4.29 & 7 \\
FEV1 (l) & 2.7 & 0.967 & 1.58 & 3.99 & 7 \\
FEVC 25-75\% (l) & 3.07 & 1.48 & 1.49 & 4.7 & 5 \\
PEF (l/min) & 4.23 & 2.531 & 1.73 & 7.57 & 4 \\
FIVC (l) & 3.34 & 0.753 & 2.38 & 4.14 & 5 \\
PIF (l/min) & 3.81 & 0.312 & 3.5 & 4.3 & 5 \\
RV (l) & 1.67 & 0.267 & 1.36 & 2 & 5 \\
MVV (l/min) & 108.5 & 38.57 & 77 & 174.2 & 5 \\
SVC (l) & 3.74 & 0.967 & 2.48 & 4.69 & 5 \\
TV (l) & 0.82 & 0.219 & 0.63 & 1.17 & 5 \\
FEV1/FVC & 0.8 & 0.113 & 0.64 & 0.93 & 7 \\
\hline
\end{tabular}

MVV, and SVC $(p<0.05)$ (Table VII). The men's equation to predict FIVC accounted for the greatest proportion of the total variance in the data. Forward stepwise regression analysis based upon the women's measured pulmonary function values yielded prediction equations for FEVC, FEV1, PEF, FIVC, PIF, RV and MVV (Table VII). All of the independent variables appeared in at least one equation. RV and MVV were inversely related to years injured, whereas FEVC was affected by injury level.

Forced stepwise regression equations for men were also developed based upon age (years), height (centimeters), weight (kilograms), years injured, and injury level
(Table VIII). Equations were not developed for women as there were insufficient data. The equation for FIVC accounts for the greatest variation in the data. Again the partial correlation for years injured remains most negatively correlated with nearly every pulmonary function variable. Age is positively correlated with some variables and negatively correlated with others.

\section{Model validations}

Models were validated by using data from randomly selected subjects, whose pulmonary function test data were not used to construct the models. One way ANOVA on 
Table IV Male wheelchair user pulmonary function percentiles

\begin{tabular}{|c|c|c|c|c|c|}
\hline & $10 \operatorname{th} \%$ & $25 \mathrm{th} \%$ & 50 th $\%$ & 75 th $\%$ & 90 th $\%$ \\
\hline \multicolumn{6}{|l|}{ Upper level injury } \\
\hline FEVC (l) & 2.78 & 3.23 & 4.1 & 4.63 & 5.48 \\
\hline FEV1 (l) & 2.27 & 2.55 & 3.18 & 3.89 & 4.59 \\
\hline FEVC $25 \%-75 \%$ & 1.43 & 2.7 & 3.60 & 4.93 & 5.17 \\
\hline PEF (l/min) & 3.2 & 4.82 & 6.41 & 7.69 & 8.26 \\
\hline FIVC (l) & 3.18 & 3.65 & 4.07 & 4.49 & 5.77 \\
\hline PIF (l/min) & 2.65 & 2.87 & 3.6 & 5.85 & 7.09 \\
\hline RV (l) & 1.14 & 1.47 & 2.25 & 2.53 & 3.03 \\
\hline $\operatorname{MVV}(1 / \min )$ & 77.4 & 97.2 & 122.7 & 157.3 & 176 \\
\hline SVC (l) & 2.88 & 3.3 & 3.9 & 4.47 & 4.84 \\
\hline TV (l) & 0.48 & 0.6 & 0.9 & 0.98 & 1.48 \\
\hline FEV1/FVC & 0.65 & 0.75 & 0.84 & 0.87 & 0.91 \\
\hline \multicolumn{6}{|l|}{ Middle level injury } \\
\hline FEVC (l) & 3.23 & 3.72 & 4.34 & 4.98 & 5.48 \\
\hline FEV1 (l) & 2.61 & 3.05 & 3.7 & 4.12 & 4.44 \\
\hline FEVC $25 \%-75 \%$ & 3.21 & 3.75 & 4.24 & 5.66 & 6.81 \\
\hline PEF (l/min) & 4.15 & 4.96 & 7.71 & 9.4 & 9.82 \\
\hline FIVC (l) & 3.5 & 4.21 & 4.79 & 5.32 & 5.79 \\
\hline PIF (l/min) & 2.85 & 3.55 & 5.22 & 7.39 & 8.74 \\
\hline RV (1) & 1.55 & 1.95 & 2.37 & 2.58 & 2.9 \\
\hline $\operatorname{MVV}(1 / \mathrm{min})$ & 98.9 & 117.6 & 149.4 & 174.6 & 201.5 \\
\hline SVC (l) & 3.3 & 3.68 & 3.93 & 4.48 & 5.15 \\
\hline TV (l) & 0.52 & 0.82 & 1.1 & 1.4 & 1.67 \\
\hline FEV1/FVC & 0.67 & 0.78 & 0.84 & 0.9 & 0.94 \\
\hline \multicolumn{6}{|l|}{ Lower level injury } \\
\hline FEVC (l) & 3.36 & 3.96 & 4.52 & 5.36 & 5.49 \\
\hline FEV1 (l) & 2.81 & 3.31 & 3.86 & 4.28 & 4.63 \\
\hline FEVC $25 \%-75 \%$ & 3.93 & 4.41 & 4.91 & 5.3 & 5.91 \\
\hline PEF (1/min) & 5.79 & 7.26 & 8.22 & 10.27 & 11.76 \\
\hline FIVC (l) & 3.52 & 4.12 & 5.13 & 5.29 & 6.04 \\
\hline $\operatorname{PIF}(\mathrm{l} / \mathrm{min})$ & 3.36 & 3.52 & 5.5 & 7.15 & 9.45 \\
\hline RV (l) & 1.24 & 1.47 & 1.8 & 2.27 & 2.8 \\
\hline $\operatorname{MVV}(1 / \min )$ & 112.4 & 134.8 & 169.6 & 190.8 & 208.4 \\
\hline SVC (l) & 3.75 & 4.14 & 4.88 & 5.15 & 5.67 \\
\hline TV (l) & 0.78 & 0.94 & 1.11 & 1.28 & 1.66 \\
\hline FEV1/FVC & 0.74 & 0.77 & 0.85 & 0.89 & 0.93 \\
\hline
\end{tabular}

each of the predicteds for prediction and validation groups showed the data were independent. In the validation group the models incorporating years of injury and level of impairment accounted for the greatest proportion of the total variance. The equations developed for women wheelchair users were not validated with the data collected.

The men's unforced generic (without years of injury and injury level) prediction equations did result in significant correlations with the validation group for $\mathrm{RV}$ $(r=0.78, p=0.005)$. The men's forced generic prediction equations also revealed significant correlations with the validation group for MVV $(r=0.47, p=0.034)$, and RV $(r=0.68, p=0.011)$. The men's unforced adapted (with years of injury and injury level) prediction equations did reveal significant correlations with the validation group for FEVC $(r=0.61, p=0.016)$, FEV1 $(r=0.69, p=0.004)$, PIF $(r=0.95$, $p=0.015), \operatorname{MVV}(r=0.59, p=0.021)$, SVC $(r=0.69, p=0.019)$, and RV $(r=$ $0.80, p=0.011)$. The men's forced adapted prediction equations did reveal significant correlations with the validation group for 
Table V Pulmonary function prediction equations for people with spinal cord injuries based upon age in years, height in centimeters, and weight in kilograms $(p<0.05)$

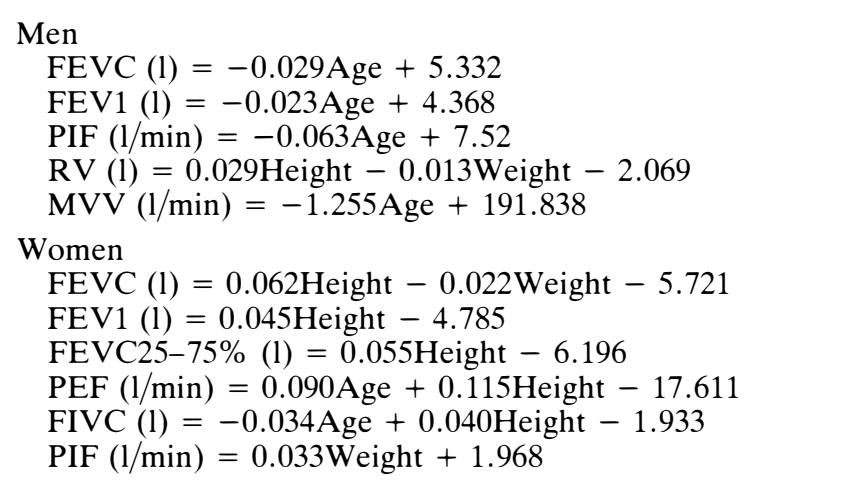

Table VI Pulmonary function prediction equations for people with spinal cord injuries based upon age, height, and weight. Equations were generated using forced stepwise regression

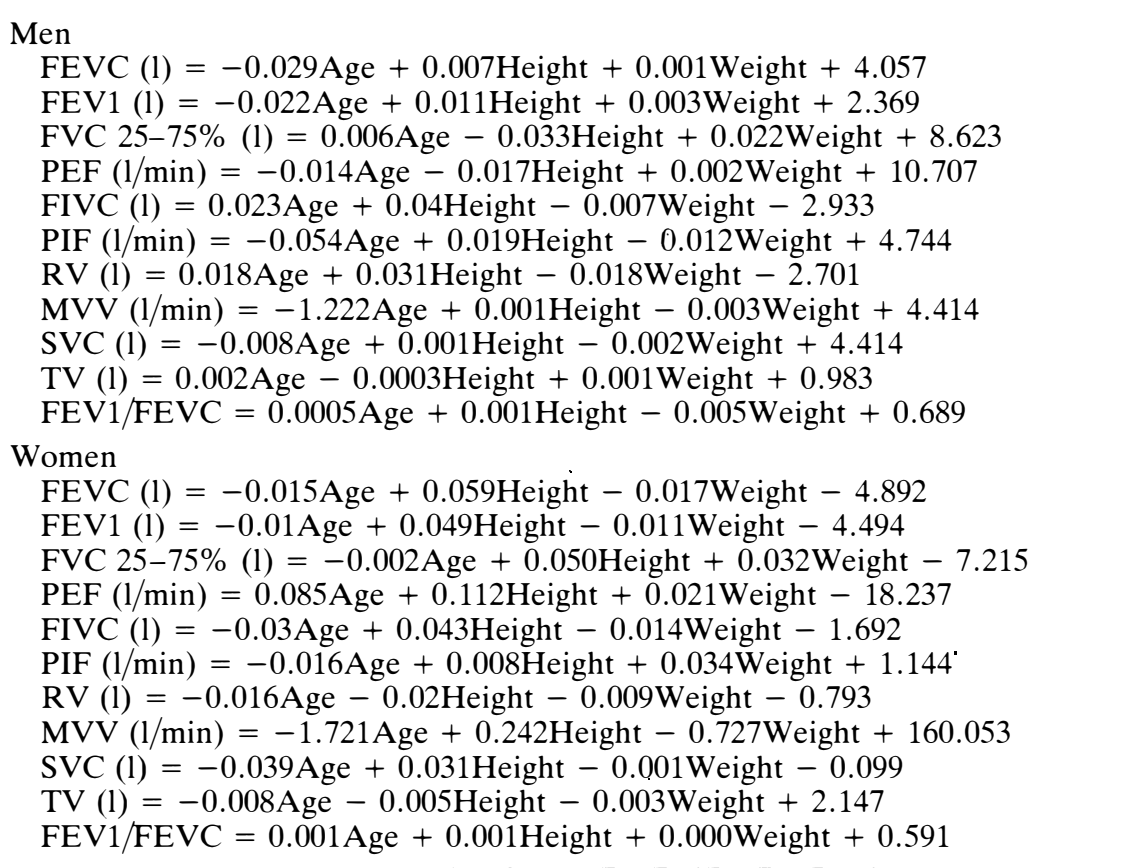

FEV1 $(r=0.61, p=0.017)$, FEV25-75\% $(r=0.84, p=0.038)$, PIF $(r=0.84, p=$ $0.078), \mathrm{MVV}(r=0.66, p=0.028), \mathrm{TV}$ $(r=0.55, p=0.078)$, and $\mathrm{RV}(r=0.56$, $p=0.036)$.

The men's adapted prediction equations were able to account for a significant portion of the variance for more pulmonary function variables in the validation group than the men's generic equations.

Comparison with prediction equations from ambulatory subject data

Pulmonary function values for women predicted by the equations developed by Crapo 
Table VII Pulmonary function prediction equations for people with spinal cord injuries based upon age, height, weight, injury level, and years of injury $(p<0.05)$

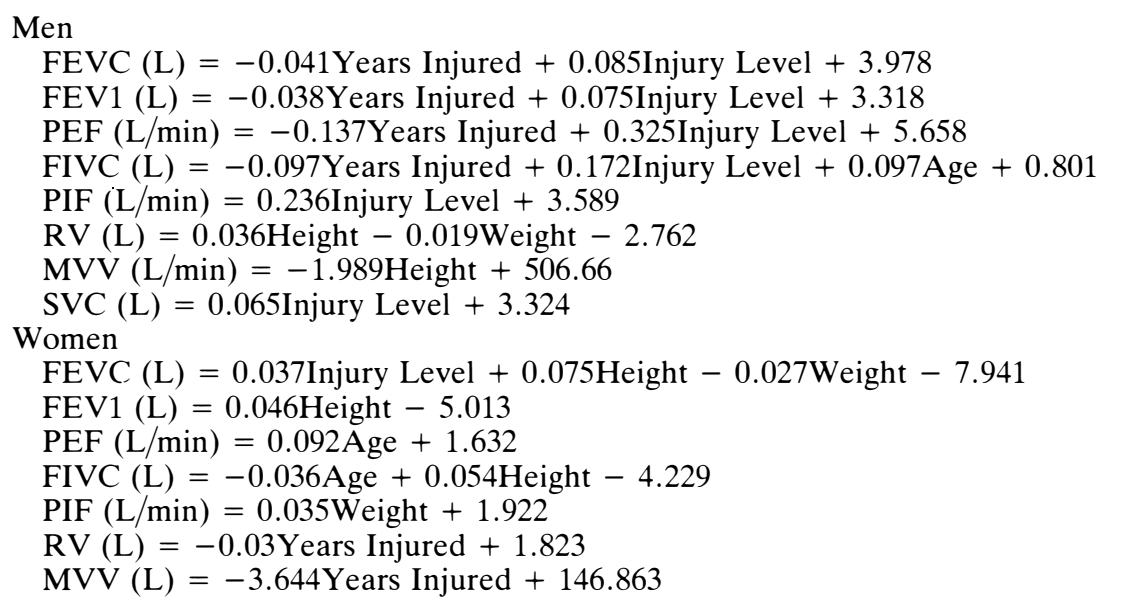

Table VIII Pulmonary function prediction equations for men with spinal cord injuries based upon age (A), height (H), weight (W), injury level (IL), and years of injury (YI). Equations were generated using forced stepwise regression

et al did yield significant correlations for FEVC $(p=0.0001)$, and FEV1 $(p=$ $0.0001)$ with those measured for the women who participated in this study. ${ }^{15-17}$ However, MVV and RV were not significantly correlated.

Correlation analysis for the men's validation group predicted, using equations developed by Crapo et al, and measured pulmonary function values showing that neither FEVC, FEV1, RV nor MVV were significantly correlated. ${ }^{15-17}$

\section{Discussion}

Pulmonary function of wheelchair users has been shown to be different from those of ambulatory people. ${ }^{2.11}$ Investigators have also reported that results predicted using ambulatory equations do not correlate well with measured results for wheelchair users. ${ }^{10,11}$ The results of the male subjects who participated in this research support these previous findings. Therefore prediction equations developed for ambulatory people may not be optimal for evaluating the pulmonary function of wheelchair users. The findings of this study indicate that for male wheelchair users time since injury and level of injury are more strongly related to pulmonary function than are age, height and weight, the typically utilized prediction variables. Furthermore, the results indicate that there are gender differences in pulmonary 
functions among wheelchairs users. The magnitude of this difference may or may not be the same as exists in the general population. Among male and female subjects pulmonary function was somewhat dependent upon level of impairment. In other words there was an inverse relationship between level of impairment and pulmonary function. This was likely related to viability of motor and sensory innervation of the primary muscles of ventilation and to better postural control.

The equations developed using the adapted independent variables accounted for a greater portion of the variance in the male validation group than did the generic independent variables. This may be because the adapted independent variables account more for the effect of physical impairment, and that the effects of physical impairment on the pulmonary system may be greater than those accounted for by simple anthropometry. The effects of aging are to some extent accounted for by years with disability. Hence, for wheelchair users years with physical impairment may contain more information related to pulmonary function than does age alone. Pulmonary function tends to decrease during natural aging. This holds true for wheelchair users as well. However, it appears to be dominated by years with physical impairment. The unforced models developed for men using the adapted prediction variables accounted for a significant portion of the variance in the measurements of the validation group for key pulmonary function parameters (FEVC, MVV, RV and SVC).

Having a mobility impairment tends to reduce pulmonary function when compared to the ambulatory population norm, and pulmonary function declines with each year since the impairment began. The original decline may be due to the loss of function associated with the physical impairment. The dependence of further decline based upon years of injury, rather than just age, suggests that other mechanisms may be in effect. One of these mechanism may be the decreased level of physical activity often observed in the wheelchair user population. Greater chronic physical activity may promote greater pulmonary function among wheelchairs users, or alter the rate of loss of pulmonary function. ${ }^{18-22}$ A close examination of Table IV indicates that some men are capable of exceeding the ambulatory population norms. These tended to be those who were more active.

\section{Conclusion}

Pulmonary functions of male wheelchair users should be predicted using equations which incorporate years with disability and level of impairment. Additional study is required to make any recommendations regarding women wheelchair users. However, study of pulmonary function of women should be made a priority. Pulmonary function of wheelchair users needs further investigation. With larger sample sizes the effects of smoking status could be investigated and included in the development of prediction equations.

\section{Acknowledgements}

This study was partially supported by funds from a California State University at Sacramento System Wide Grant, United States Olympic Foundation Contracts (U91-055-E-NW and U92-026-E-NW), the National Wheelchair Athletic Association, the US Department of Veterans Affairs, and the Rehabilitation Services Administration, US Department of Education (\#H129E00005). The authors thank Annette Vosse, Darla Jones, Sigrid Wagner, Kim Asato, Rosi Cooper and Jim Ster III for their assistance. The authors are very grateful for the voluntary participation of the many people who served as subjects in this study.

\section{References}

1 Wells CL, Hooker SP (1990) The spinal injured athlete. Adapted Phys Activity Q 7: 265-285.

2 Van Loan MD, Mc Cluer MD, Loftin JM, Boileau RA (1987) Comparison of physiological responses to maximal arm exercise among able-bodied, paraplegics and quadriplegics. Paraplegia 25: 397-405.

3 Shephard R (1988) Sports medicine and the wheelchair athlete. Sports Med 4: 226-247. 
4 Cole TM and Edgerton VR (1990) Report of the task force on medical rehabilitation research. National Institute of Health, Hunt Valley, Maryland.

5 Leaf DA, Adkins RH, Greenwood J, Bahl RA (1990) Maximal aerobic capacity and theoretical implications for longevity revisited in spinal cord injury patients. Ann Sports Med 5: 133-137.

6 Zwiren LD, Bar-Or O (1974) Responses to exercise of paraplegics who differ in conditioning level. Med Sci Sport 7(2): 94-98.

7 Ferrara MS, Buckley WE, McCann BC, Limbird TJ, Powell JW, Robl R (1992) The injury experience of the competitive athlete with a disability: prevention implications. Med Sci Sports Exerc 24(2): 184-188.

8 Hulleman KD, List M, Matthes D (1975) Spiroergometric and telemetric investigations during the XXI International Stoke Mandeville Games 1972 in Heidelberg. Paraplegia 13: 109-123.

9 Kokkola K, Moller K, Lehtonen T (1975) Pulmonary function in tetraplegic and paraplegic. Ann Clin Res 7: 76-79.

10 Ohry A, Molho M, Rozin R (1975) Alterations of pulmonary function in spinal cord injured patients. Paraplegia 13: 101-108.

11 Stone D and Keltz H (1964) The effect of respiratory muscle dysfunction on pulmonary function. J Appl Physiol 43: 621-629.

12 Gross D, Ladd HW, Riley EJ, Macklem PT, Grassino A (1980) The effect of training on strength and endurance of the diaphragm in quadriplegia. Am J Med 68: 27-35.

13 Cherniack RM (1977) Pulmonary Function Testing. W B Saunders Co, Philadelphia, PA.

14 Miles DS, Sawka MN, Wilde SW, Durbin RJ, Gotshall RW, Glaser RM (1982) Pulmonary function changes in wheelchair athletes subsequent to exercise training. Ergonomics 25(3): 239-246.

15 Crapo RO, Morris AH, Gardner RM (1981) Reference spirometric values using techniques and equipment that meet ATS recommendations. Am Rev Respir Dis 123: 659-664.

16 Crapo RO, Morris AH (1981) Standardized single breath normal values for carbon monoxide diffusing capacity. Am Rev Respir Dis 123: 185-189.

17 Crapo RO, Morris AH, Clayton PD, Nixon CR (1982) Lung volumes in healthy non-smoking adults. Bull Eur Physiopathol Respir 18: 419-425.

18 Cooper RA (1990) A comparison of pulmonary functions of wheelchair racers in their racing and standard wheelchairs. RESNA 14th Annual Conference Kansas City, MO: 245-247.

19 Cooper RA, Baldini FD, Robertson RN, Jones D, Monical S, Vosse A (1992) Pulmonary function of elite wheelchair athletes. Palaestra 8(4): 10.

20 Aronson RM, Carley DW, Onal E, Wilborn J, Lopata M (1991) Upper airway muscle activity and the thoracic volume dependence of upper airway resistance. J Appl Physiol 70(1): 430-438.

21 Leith DE, Bradley M (1976) Ventilatory muscle strength and endurance training. J Appl Physiol 41(4): 508-516.

22 Powers SK, Lawler J, Criswell D, Lileu F, Martin D (1992) Aging and respiratory muscle metabolic plasticity: effects of endurance training. J Appl Physiol 72(3): 1068-1073. 